\title{
Scrotal Filariasis, the Importance of Filarial Dance Sign in Scrotal Ultrasound
}

\author{
Mohsen Kamel Arid \\ Radiology Department, Benha Faculty of Medicine, Benha, Egypt \\ Email: mkarid2002@yahoo.com
}

How to cite this paper: Arid, M.K. (2021) Scrotal Filariasis, the Importance of Filarial Dance Sign in Scrotal Ultrasound. Open Journal of Medical Imaging, 11, 1-5. https://doi.org/10.4236/ojmi.2021.111001

Received: November 22, 2020

Accepted: January 24, 2021

Published: January 27, 2021

Copyright (C) 2021 by author(s) and Scientific Research Publishing Inc. This work is licensed under the Creative Commons Attribution International License (CC BY 4.0).

http://creativecommons.org/licenses/by/4.0/

\begin{abstract}
Objective: To study the value of the filarial dance sign as a diagnostic sign in scrotal ultrasonography in human Bancroftian filariasis. Methods: We studied 3 patients with scrotal pain, with high-resolution ultrasonography (HRUS) of scrotum including power and color Doppler study. All patients underwent peripheral blood smear. Results: Multiple cystic spaces containing motile echogenic twisted tubular structures were observed in the 3 patients. Peripheral blood smears in the 3 patients confirmed the presence of microfilaria. Mild hydrocele in two patients. Conclusions: HRUS is a useful technique for diagnosing scrotal filariasis in symptomatic patients and is very useful in the follow-up treatment.
\end{abstract}

\section{Keywords}

Filaria Dance Sign (FDS), HRUS of Scrotum, Scrotal Filariasis, Wuchereria Bancrofti

\section{Background}

Lymphatic filariasis is a clinical syndrome caused by the worms Wuchereria bancrofti, Brugia malayi and Brugia timori. The most common clinical form of the disease is hydrocele, with lymphedema and elephantiasis occurring less commonly [1].

After copulation, the female adult worm releases a large number of live microfilariae into the lymphatic vessels and ultimately finds their way into the bloodstream. They circulate in the blood in the largest numbers at night and are said to have nocturnal periodicity [2]. Female mosquitoes of the Culex, Aedes, and Anopheles genera act as intermediate hosts. The mosquitoes ingest the microfilariae during a blood meal. The adult worms can live for 15 years [2]. 
Adult worms of $\mathrm{W}$. bancrofti commonly reside in the lymphatics. The female worms measure 80 to $100 \mathrm{~mm}$ in length and 0.24 to $0.30 \mathrm{~mm}$ in diameter, while the males measure about $40 \mathrm{~mm}$ by $1 \mathrm{~mm}$. Adults produce microfilariae measuring 244 to $296 \mu \mathrm{m}$ by 7.5 to $10 \mu \mathrm{m}$, which are sheathed and have nocturnal periodicity [3].

Lymphatic filariasis affects over 120 million people throughout the tropics and sub-tropics of Asia, Africa, the Pacific, and the Caribbean and South America [3].

Diagnosis of the disorder is based on the appearance of microfilariae in blood smears. Serologic tests may be of some value [4].

Diethylcarbamazine (DEC) is the drug of choice. The drug kills the microfilariae and some of the adult worms, by 12 -day treatment of DEC $(6 \mathrm{mg} / \mathrm{kg} /$ day $)$. Also, one day treatment is generally as effective as the 12-day regimen. DEC is generally well tolerated with limited Side effects [3].

\section{Methods}

We studied 3 patients with scrotal pain, mainly to exclude varicocele in whom the filarial dance sign was demonstrated on high-resolution ultrasonography (HRUS), during the period from November 2011 to November 2019, during my work as radiologist in private hospital in Abu Dhabi. The age of male patients, between 25 to 30 year, Median age $=27.6$, mainly Indian decent (endemic areas). All patients underwent peripheral blood smear at night.

\section{Results}

Multiple cystic spaces containing motile echogenic twisted tubular structures were observed in the 3 patients. Two patients have multiple channels, one had a single space surrounded by a network of small of dilated channels with absence of blood flow in color and power doppler interrogation of these spaces, thus varicocele was excluded as a cause. Mild hydrocele was demonstrated in two patients, these HRUS findings were demonstrated in the right scrotum at neck of the testis at junction epididymis and spermatic cord, there was no scrotal swelling or scrotal enlargement or edema, no inguinal hernia or lymphadenopathy.

Peripheral blood smears revealed that all 3 patients had microfilaremia. The geometric mean microfilarial density was $268 \mathrm{c} / \mathrm{mL}$ among those with unilateral filaria dance signs.

\section{Supporting Material}

Figure 1, Figure 2 and Figure 3 demonstrate this sign for 3 different patients, 3 short videos for this sign can be reached on: AridRad.blogspot.com.

\section{Discussion}

Filariasis may affect the scrotal lymphatic channels, causing epididymitis, hydrocele or scrotal swelling [5]. 


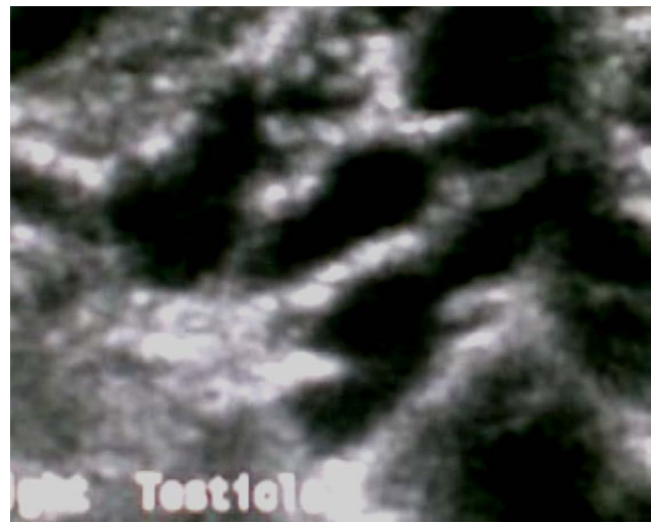

Figure 1. Collection of small moving echogenic foci inside the dilated lymphatic channels at the right testis (FDS).

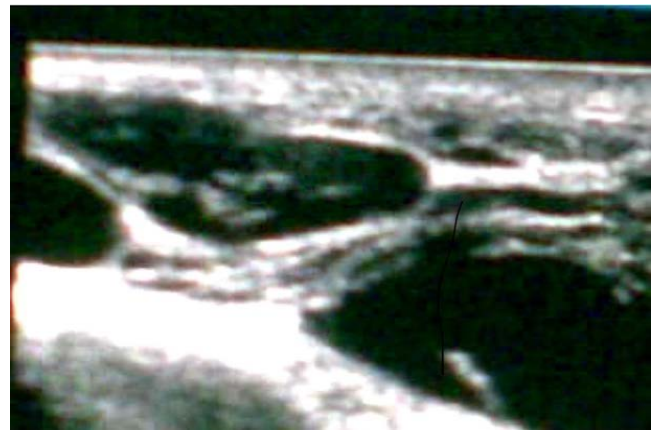

Figure 2. A twirling motion of microfilariae (W. bancrofti) was demonstrated in dilated lymphatic channels.

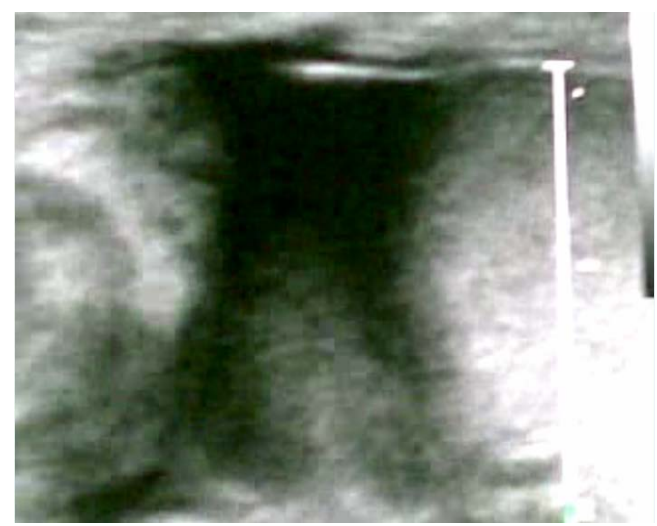

Figure 3. Mild right hydrocele is noted in a patient with Scrotal filariasis.

The FDS was first described by Amaral and coworkers in 1994 [6]. They described the movements of live adult filarial worms in the lymphatic vessels as on HRUS as linear echogenic structures with persistent, random, almost tireless twirling movements. This sign is so striking that once it is detected on HRUS, it cannot be mistaken for anything else [6]. 
However, the encounter of very similar sonographic findings in nonendemic areas for filariasis and in men with negative blood findings for filariasis [5] makes this sign not specific for Filaria infection only.

The majority of patients in nonendemic areas had a history of vasectomy or, less frequent, bacterial epididymitis. The unifying feature of men with "filarial dance" in endemic and nonendemic areas appeared to be the presence of a process causing spermatic duct obstruction. A post-mortem study on the epididymides in men with spermatic duct obstruction demonstrated dilated epididymal tubules containing large clumps of agglutinated spermatozoa [5].

The "filaria dance sign (FDS)" suggesting the presence of adult worms was observed in 22 carriers (61\%). The preferential site of location of the adult worms was the intrascrotal juxtatesticular lymphatic vessels in "nests" along the lymphatic vessels of the epididymis, spermatic cord and paratesticular region. The number of nests varied between 1 and 4 . In 95\% of cases, localization of the worms was unilateral [7].

If the patient has FDS in endemic area and microfilaria demonstrated in peripheral blood smear, it is diagnostic of active filariasis infection. If the patient has FDS in non endemic area it denotes epididymal obstruction, and in these patients microfilaria not demonstrated in peripheral blood film. The floating twisty dancing echogenic bodies in endemic area are adult filiaria worms, in non edemic ares are of moving, hyperechoic particles within cystic dilatations of an enlarged epididymis in a patient with spermatic duct obstruction. The particles most likely represent clusters of agglutinated spermatozoa [5]. In our study, we saw sizeable moving tubular structures usually lodged in one lymphatic space. This sign was demonstrated on right side only in our study, at the epididymis. The continuous swirling fast movement is striking in scrotal filariasis, researchers had done needle aspiration [8] for these patients they found, the aspirate contained microfilaria, because the needle cannot aspirate the whole worm which is larger than the bore of the needle.

The spaces seen in spermatic cord obstruction are dilated microtubules of epididymis containing small nearly equal size small echogenic oscillating foci. The spaces in filariasis are dilated lymphatic channels containing relatively large curved moving structures that are adult worms of filariasis. The spaces are scatterd in filariasis but in epididymis obstruction are grouped together.

The presence of microfilaria in blood smear in these patients should prompt treatment with diethylcarbamazine drug therapy and follow up is advised.

\section{Conclusion}

Patients with FDS in endemic areas are due to lymphatic obstruction, and microfilaria demonstrated in blood smear is diagnostic for Scrotal Filariasis. It is also demonstrated in patients who have epididymal obstruction.

\section{Conflicts of Interest}

The author declares no conflicts of interest regarding the publication of this paper. 


\section{References}

[1] World Health Organization (2010) World Health Organization Global Programme to Eliminate Lymphatic Filariasis. Progress Report 2000-2009 and Strategic Plan 2010-2020. Geneva, Switzerland.

[2] Weatherly, N.F. (1998) Medical helminthology. In: Willet, J. and Wilfret, A., Eds., Zinsser's Microbiology, 19th Edition, Appleton \& Lange, Norwalk, 984-986.

[3] CDC Centers for Disease Control and Prevention Web Site. Lymphatic Filariasis, Epidemiology and Risk Factors, Diagnosis and Treatment. https://www.cdc.gov/parasites/lymphaticfilariasis/epi.html

[4] Grove, D.I. (2000) Tissue Nematodes. In: Mandell, G.L., Benett, J.E. and Dolin, R., Eds., Principles and Practice of Infectious Diseases, 5th Edition, Churchill Livingstone, London, 246-247. https://doi.org/10.1097/00024382-200014020-00033

[5] Leenknegt, B., Diss, L. and Sidhu, P.S. (2019) Dancing Megasperm. Journal of the Belgian Society of Radiology, 103, 5. http://doi.org/10.5334/jbsr.1687

[6] Amaral, F., Dreyer, G., Figueredo-Silva, J., et al. (1994) Live Adult Worms Detected by Ultrasonography in Human Bancroftian Filariasis. The American Journal of Tropical Medicine and Hygiene, 50, 753-757. https://doi.org/10.4269/ajtmh.1994.50.753

[7] Reddy, G.S., Das, L.K. and Pani, S.P. (2004) The Preferential Site of Adult Wuchereria Bancrofti: An Ultrasound Study of Male Asymptomatic Microfilaria Carriers in Pondicherry, India. The National Medical Journal of India, 17, 195-196.

[8] Nitin, G., Chaubal, M.D., Gautam M.P.D., Jyoti, N.C. and Subhash, K.R. (2003) Dance of Live Adult Filarial Worms is a Reliable Sign of Scrotal Filarial Infection. Journal of Ultrasound in Medicine, 22, 765-769.

https://doi.org/10.7863/jum.2003.22.8.765 